\title{
Dinámica estacional de la abundancia de piquituerto común Loxia curvirostra L., 1756 en dos localidades del Pirineo Navarro e implicaciones para su seguimiento mediante anillamiento.
}

\section{Seasonal abundance patterns of common cross- bills Loxia curvirostra L., 1756 in two localities of the Navarran Pyrenees and implications for its survey through ringing.}

Daniel Alonso', Juan Arizaga ${ }^{1 *}$

\section{\%}

\section{Resumen}

Las estaciones de anillamiento de aves de esfuerzo constante realizan un papel clave en el seguimiento de poblaciones a largo plazo. El periodo de muestreo de este tipo de estaciones se ajusta a la época de nidificación de las especies más abundantes, en el caso de España entre los meses de abril-mayo y agosto. En consecuencia, aquellas especies cuya reproducción no coincida con la del grueso de especies quedan, invariablemente, poco o indebidamente representadas en este tipo de programas de seguimiento. Lo mismo ocurre con especies que, debido a su comportamiento, requieren de técnicas de muestreo especiales o específicas para su monitorización. Ejemplo de tales excepciones sería el piquituerto común Loxia curvirostra, L. 1758. En este artículo se describe el patrón estacional de la abundancia de piquituertos en dos localidades del Pirineo Navarro empleando datos de anillamiento obtenidos durante un periodo de 22 años. Los patrones observados fueron homogéneos entre ambos puntos de muestreo. Nuestros resultados sugieren que el promedio de capturas entre los meses de enero y abril podría utilizarse como un índice de la abundancia de individuos adultos durante el periodo de cría en el Pirineo Occidental.

Palabras clave: Anillamiento, aves forestales, Bigüézal, fenología, pinares, Uztárroz.

\footnotetext{
1 Departamento de Ornitología, Sociedad de Ciencias Aranzadi

Zorroagagaina 11, 20014 Donostia
} 


\begin{abstract}
Constant effort bird ringing stations develop a relevant role in long-term population monitoring programs. The sampling period in these stations coincides with the most abundant species' breeding period; in Spain this lasts from April or May to August. Thus, those birds breeding in other periods would remain poorly represented in this type of programs. This is also applicable to species needing very specific sampling techniques. The common crossbill Loxia curvirostra, L. 1758 is an example of this type of species. The aim of this paper is to describe the seasonal abundance patterns of crossbills in the Pyrenees in order to find a useful method to estimate long-term population trendsusing data obtained during a 22-year ringing program at two localities in the Pyrenean region of Navarre. The patterns observed were homogeneous in the two sampling localities. The mean number of captures from January to April could be used as an index of abundance of adult breeding birds.
\end{abstract}

Key words: Ringing, forest-dwelling bird species, Bigüézal, phenology, pine forest, Uztárroz.

\title{
Laburpena
}

Esfortzu konstanteko hegaztien eraztuntze guneek zeregin garrantzitsua dute epe luzeko populazioen jarraipenean. Mota horretako guneetako laginketa bat etortzen da espezie ugarienen ugaltze garaiarekin eta Espainian apiril-maiatza eta abuztu artean izaten da hori. Ondorioz, beraien ugalketa espezie gehienen ugaltze garaiarekin batera ez duten espezieak behin eta berriz geratzen dira gutxi edo gaizki ordezkatuta. Berdin gertatzen da beren monitorizaziorako laginketa teknika bereziak edo espezifikoak behar dituzten espezieen kasuan. Salbuespen horien adibide da mokoker arrunta Loxia curvirostra, L. 1758. Bada, artikulu honetan mokoker arrunten garai desberdinetako ugaritasun patroia deskribatzen da, 22 urtean Nafarroako Pirinioetako bi herrixkatan jasotako eraztunketa datuak erabilita lortutakoa. Bi laginketa puntuetan behatutako patroiak bata bestearekiko homogenoak izan ziren. Gure emaitzek iradokitzen dutena da urtarril eta apiril arteko harrapaketen batezbestekoa erabil litekeela ale helduen ugaritasun indizetzat Ekialdeko Pirineoetako ugaltze sasoian.

Gako hitzak: eraztunketa, basoko hegaztiak, Biguezal, fenología, pinares, Uztarrotz.

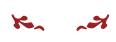

\section{Introducción}

Las estaciones de anillamiento de aves de esfuerzo constante realizan un papel clave en el seguimiento de poblaciones a largo plazo (Ralph \& Dunn, 2004). Tales estaciones tienen como último objetivo la estimación de parámetros demográficos de las especies más frecuentemente capturadas, generalmente paseriformes, entre ellos el tamaño y tendencia de la población, productividad y supervivencia (Peach et al., 1996; Peach et al.1998; Nur et al., 2000). Un índice de abundancia es aquel capaz de establecer un valor de abundancia estandarizado para un esfuerzo y periodo de muestreo dados en un lugar y unidad de 
tiempo determinados, que por tanto pueda ser comparado con valores que se obtengan en ese mismo u otros lugares (Peach et al., 1996). El periodo de muestreo de este tipo de estaciones se ajusta a la época de nidificación de las especies más abundantes, en el caso de España entre los meses de abril o mayo y agosto (e.g., Arizaga et al., 2013). En consecuencia, aquellas especies cuya reproducción no coincida con la del grueso de especies quedan invariablemente poco o indebidamente representadas en este tipo de programas de seguimiento. Lo mismo ocurre con especies que, debido a su comportamiento, requieren de técnicas de muestreo especiales o específicas para su monitorización.

Ejemplo de tales excepciones sería el piquituerto común Loxia curvirostra, L. 1758. El piquituerto es un paseriforme ampliamente distribuido en el Paleártico (Cramp \& Perrins, 1994). En España cría principalmente en Pirineos, Sistema Ibérico y Sistema Central, así como las sierras del sur y este peninsular, incluido el archipiélago balear (Borrás \& Senar, 2003). Adaptado a la disponibilidad de semillas de coníferas, el piquituerto se comporta como un reproductor oportunista (Newton, 1972). Muchas poblaciones crían en pleno invierno y pueden presentar, adicionalmente, segundas puestas en verano (Alonso \& Arizaga, 2011). Además, la especie explota el estrato más alto del bosque, por lo que su captura es difícil y está condicionada, normalmente, a la existencia de puntos de agua o concentración de sales en el suelo (Alonso \& Arizaga, 2011). Estas particularidades hacen que tanto el periodo como la técnica de muestreo utilizados en las estaciones de anillamiento para la monitorización de aves nidificantes sean inadecuados para el piquituerto.

Las poblaciones ibéricas de piquituerto son sedentarias, debido a la estabilidad de la productividad de las coníferas del sur de Europa (Senar et al., 1993). En determinadas regiones, como el Pirineo Occidental, parece existir cierta variabilidad en el patrón estacional de abundancia a nivel local (D. Alonso, obs. pers.). Esta variación podría estar relacionada tanto con movimientos entre diferentes zonas a nivel local como con el aporte de aves nórdicas o individuos procedentes de otras zonas de España en determinadas épocas del año (Arizaga et al., 2014; Arizaga et al., 2015).

El patrón estacional de abundancia de poblaciones de piquituerto en España ha sido muy poco estudiado y se limita básicamente a trabajos de censos de aves forestales a lo largo de un ciclo anual (Purroy, 1975; Sánchez, 1991). Los objetivos de este artículo son: 1) Describir el patrón estacional de abundancia de piquituertos en bosques de pino silvestre del Pirineo Occidental; y, 2) Determinar un periodo de muestreo adecuado para estimar parámetros poblacionales a partir de datos obtenidos mediante anillamiento científico.

\section{Material y métodos}

El estudio se realizó en dos localidades del Pirineo Navarro separadas una distancia de 25

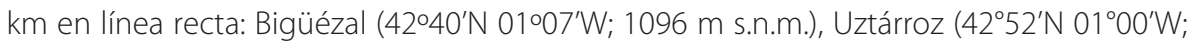
1383 m s.n.m.) (Fig. 1). En ambas localidades dominan los bosques maduros de pino silvestre (Loidi \& Báscones, 2006). 


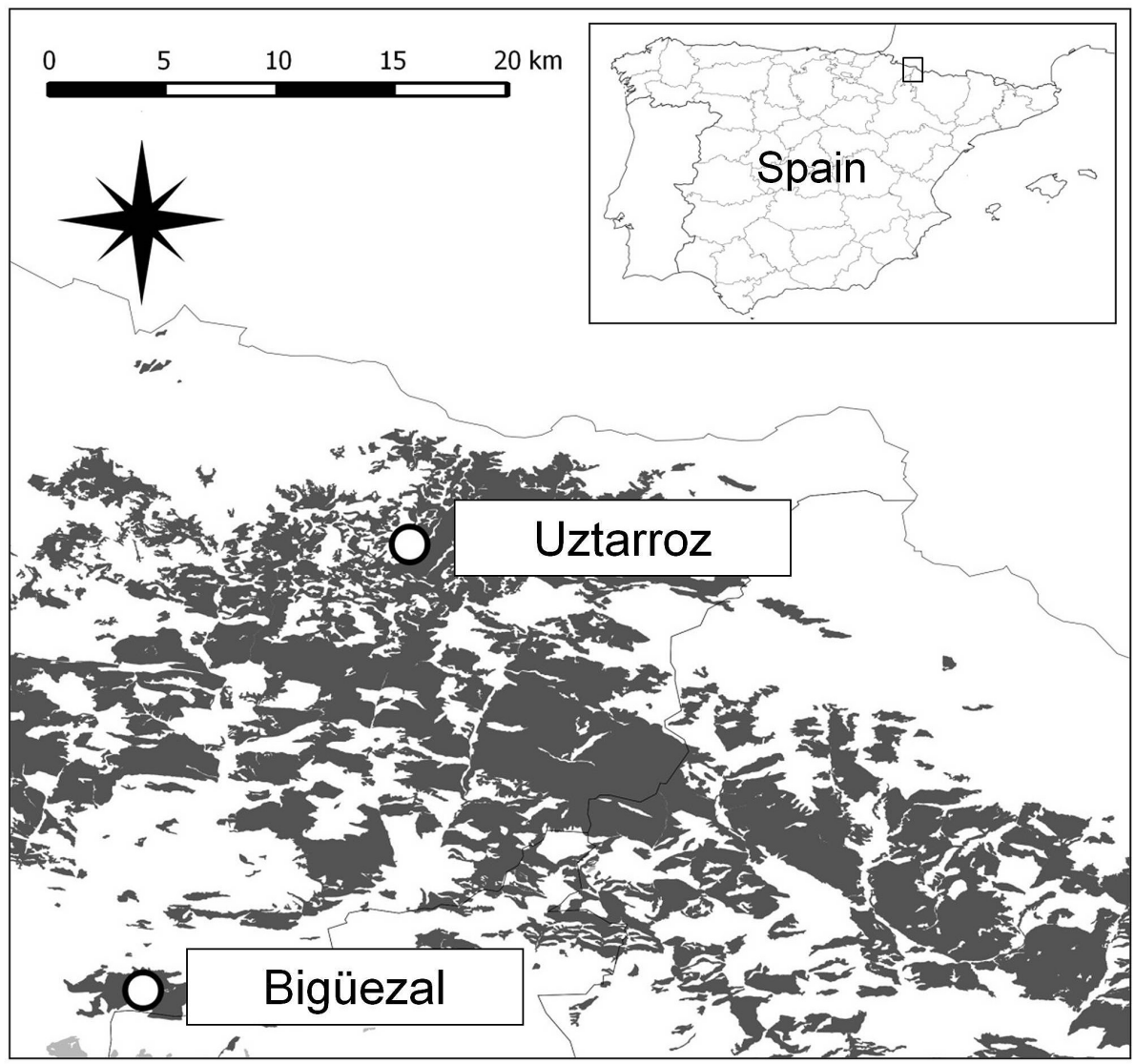

Fig. 1.- Puntos de muestreo de piquituertos en el Pirineo Navarro. La superficie en gris indica la distribución de pino silvestre.

Fig. 1.- Common crossbills sampling localities in the Pyrenean region of Navarre. The shadowed area in grey shows the Scots pine distribution range.

El periodo de estudio abarcó un total de 22 años, desde 1993 hasta 2014. Durante este periodo, se muestreó un mínimo de una vez por quincena, si bien, en ocasiones y debido a causas diversas (principalmente, meteorología adversa), el número de visitas se redujo a una por mes. En total se llevaron a cabo 619 jornadas de muestreo (Uztárroz: 285 días; Bigüezal: 334 días).

Para la captura se utilizaron redes de niebla emplazadas en zonas donde los piquituertos acuden para alimentarse de minerales. En cada zona se utilizó siempre un número fijo de metros de red situadas en los mismos puntos (12 m en Uztárroz y 24 m en Bigüézal). Las capturas se llevaron a cabo durante un periodo de $4 \mathrm{~h}$ a partir de la salida del sol.

Cada ejemplar fue anillado con una anilla de aluminio para su reconocimiento individual; en caso de recaptura se anotó el número de anilla. El sexo y la edad fueron determinados 
a través del examen del plumaje (Svensson, 1996). Se consideraron dos categorías de edad: jóvenes y adultos. Al primer grupo fueron asignadas aves de plumaje totalmente juvenil (código de edad EURING: 3J) o capturadas tras una muda parcial realizada durante el año de nacimiento (código de edad EURING: 3); al segundo se asignaron las aves con dos o más años de vida (código de edad EURING: 4, 5, o 6). En el caso de hembras adultas se examinó adicionalmente la zona ventral para determinar la existencia de placa de incubación, cuyo desarrollo se midió según una escala con 5 niveles: 0, ausencia de placa; 1, en desarrollo incipiente; 2, casi completamente desarrollada; 3, completamente desarrollada; 4, en regresión; 5, plumas en desarrollo (Pinilla, 2000). Las categorías 2 y 3 se corresponden principalmente con el periodo de incubación.

En total se realizaron 6.747 capturas, de las cuales 5.445 correspondieron a individuos distintos y 1.302 (23,9\%) a recapturas (Bigüezal; jóvenes, 958, adultos, 1.987; Uztárroz: jóvenes, 692, adultos, 3.110). Cada ejemplar fue considerado una sola vez por día de muestreo.

\section{Análisis estadísticos}

Para determinar si el número de capturas varió mensualmente según la zona y categoría de edad llevamos a cabo un modelo lineal generalizado con el número promedio de capturas por mes, año y zona (esto es, para cada mes y año, se calculó en cada zona la media de capturas) como variable objeto y el mes como factor fijo. El año no fue incluido como factor aleatorio debido al limitado tamaño muestral. El modelo lineal generalizado se realizó para adultos y jóvenes y para cada zona de muestreo separadamente. Debido a la distribución de errores de la variable objeto se empleó un modelo log-lineal con distribución de errores Gamma.

Para determinar hasta qué punto la variabilidad interanual de la abundancia se ajusta a procesos que operan a escala regional o bien a determinantes de carácter local se llevó a cabo un test de correlación de la abundancia entre ambos puntos de muestreo. Para ello calculamos el promedio de capturas de adultos por año (meses de enero a abril; para más detalles ver resultados) y zona.

Para determinar el porcentaje de hembras en periodo de incubación se consideraron aquellas con placas con código 2 y 3 en relación al total de hembras adultas capturadas por mes.

Los análisis estadísticos se llevaron a cabo mediante el programa R (R Core Team, 2014) y el paquete "Ime4" (Bates et al., 2014).

\section{Resultados}

Tanto para Bigüézal como para Uztárroz, el número diario de capturas de aves adultas varió significativamente entre meses (Tabla 1). En Bigüézal, el número de capturas de adultos se 


\begin{tabular}{|lccc:cccc|}
\hline & \multicolumn{3}{c|}{ Bigüézal } & \multicolumn{3}{c|}{ Uztárroz } \\
\hline Factor & $B$ & $\mathrm{SE}(B)$ & $P$ & $B$ & $\mathrm{SE}(B)$ & $P$ \\
\hline Intercepto & $+0,10$ & 0,02 & $<0,001$ & $+0,05$ & 0,02 & 0,002 \\
Feb1 & $-0,01$ & 0,03 & 0,619 & $-0,01$ & 0,02 & 0,880 \\
Mar & $-0,02$ & 0,03 & 0,496 & $-0,01$ & 0,02 & 0,851 \\
Abr & $+0,01$ & 0,03 & 0,830 & $-0,01$ & 0,02 & 0,661 \\
May & $+0,05$ & 0,04 & 0,213 & $+0,09$ & 0,03 & 0,001 \\
Jun & $+0,35$ & 0,13 & 0,006 & $+0,25$ & 0,07 & $<0,001$ \\
Jul & $+0,15$ & 0,05 & 0,003 & $+0,22$ & 0,06 & $<0,001$ \\
Ago & $+0,18$ & 0,05 & 0,001 & $+0,10$ & 0,03 & 0,003 \\
Sep & $+0,27$ & 0,08 & $<0,001$ & $+0,17$ & 0,04 & $<0,001$ \\
Oct & $+0,25$ & 0,07 & 0,001 & $+0,17$ & 0,05 & 0,001 \\
Nov & $+0,14$ & 0,06 & 0,025 & $+0,09$ & 0,03 & 0,008 \\
Dic & $+0,03$ & 0,04 & 0,449 & $+0,11$ & 0,04 & 0,012 \\
\hline
\end{tabular}

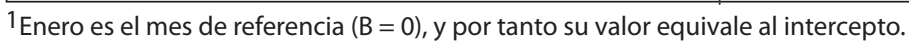

Tabla 1.- $B$-parámetros del efecto del mes en el número de capturas de piquituertos adultos capturados en dos localidades del Pirineo Navarro ( $n=619$ días de muestreo).

1 January is the reference month $(B=0)$ and therefore its value is the equivalent of the intercept

Table 1.- $B$-parameter estimates of the effect of month on the number of captures of adults caught at two localities of the Navarran Pyrenees ( $n=619$ sampling days).

mantuvo estadísticamente similar (y máximo) entre los meses de diciembre y mayo, momento a partir del cual descendieron hasta alcanzar un mínimo en junio. Las capturas aumentaron nuevamente en julio y volvieron a reducirse paulatinamente hasta el mes de septiembre. De octubre a noviembre, el número de capturas se incrementó hasta alcanzar otro máximo en diciembre (Tabla 1; Fig. 2). En Uztárroz ocurrió algo similar. El máximo número de capturas tuvo lugar entre los meses de enero y abril. A partir de entonces las capturas disminuyeron significativamente, con dos leves picos en agosto y noviembre (Fig. 2).

No se registró una correlación significativa en el número diario de capturas de aves adultas entre las dos zonas de muestreo $(r=0,041, P=0,871, n=18)$.

El número diario de capturas de aves jóvenes sólo varió significativamente entre meses en Bigüezal (Tabla 2). En esta zona, las capturas alcanzaron un máximo en julio y otro en diciembre (sin diferencias significativas entre ambos meses). Durante el resto del año, el número de capturas fue reducido (Fig. 2).

Entre los meses de enero y mayo hallamos un porcentaje de hembras adultas más alto que en el periodo de incubación en ambas zonas de muestreo. El máximo se alcanzó en marzo, 

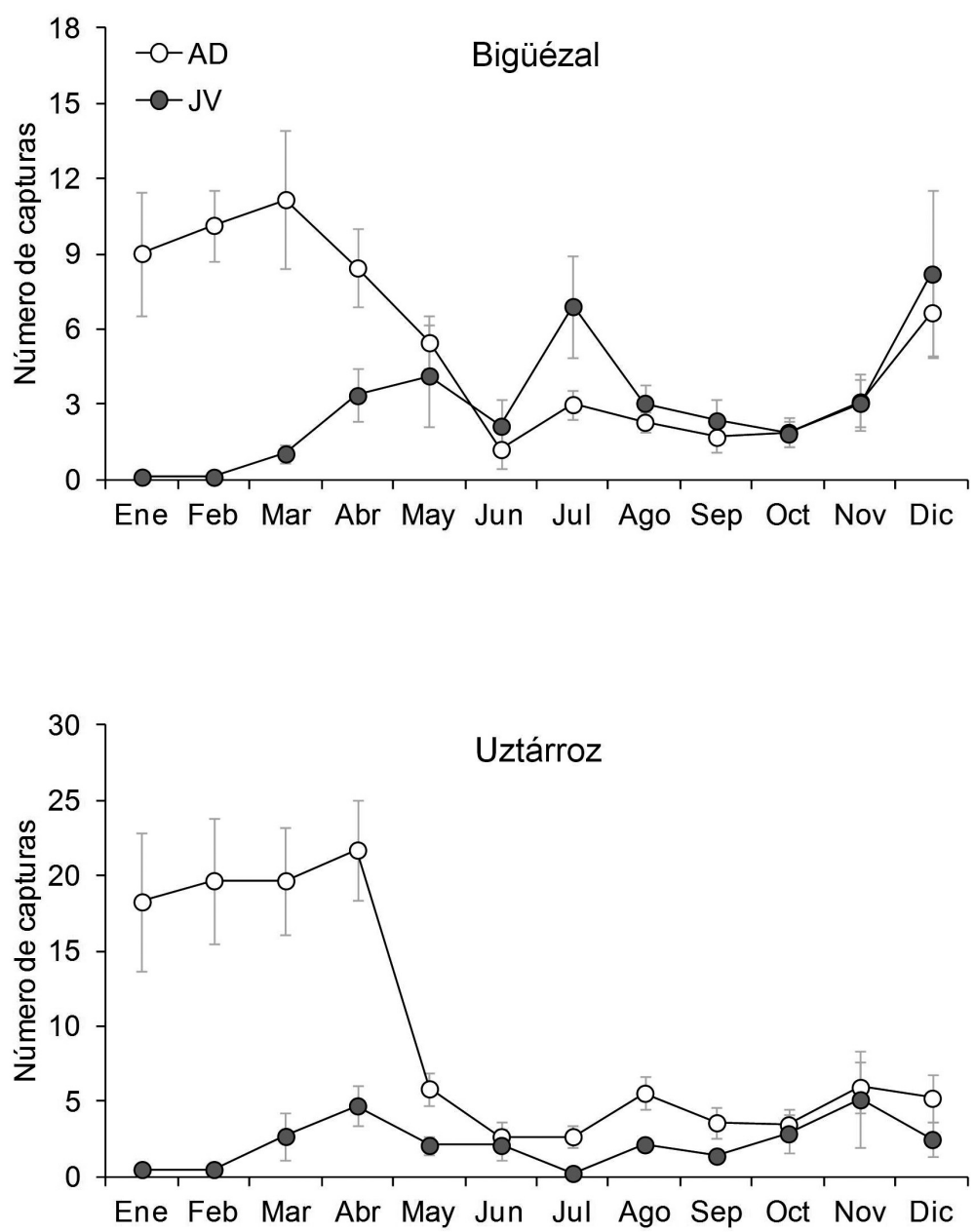

Fig. 2.- Número promedio ( $\pm \mathrm{EE}$ ) de capturas (por día) en los dos puntos de muestreo en el Pirineo Navarro (tamaño muestral: Bigüezal, 334 días; Uztarroz, 285 días).

Fig. 2.- Mean $( \pm \mathrm{SE})$ number of captures (per day) at the two sites where the crossbills were captured in the Pyrenean region of Navarre (sample size: Bigüezal, 334 days; Uztarroz, 285 days).

con aproximadamente un 50\% de hembras en reproducción activa (Fig. 3). Entre los meses de julio y diciembre se capturaron también algunos ejemplares reproductores en ambas zonas. En Uztárroz, observamos otro pico importante en el mes de agosto, en el cual algo más del $40 \%$ de las hembras capturadas presentaron placa incubatriz activa.

El porcentaje de hembras en estado reproductor capturadas en Uztárroz fue invariablemente más alto que en Bigüezal (Fig. 3). 


\begin{tabular}{|lccc:cccc|}
\hline & \multicolumn{3}{c}{ Bigüézal } & \multicolumn{3}{c|}{ Uztárroz } \\
\hline Factor & $B$ & $\mathrm{SE}(B)$ & $P$ & $B$ & $\mathrm{SE}(B)$ & $P$ \\
\hline Intercepto & $+0,89$ & 0,23 & $<0,001$ & $+0,67$ & 0,29 & 0,024 \\
Feb1 & $+0,03$ & 0,31 & 0,933 & $+0,09$ & 0,37 & 0,808 \\
Mar & $-0,41$ & 0,25 & 0,103 & $-0,41$ & 0,30 & 0,172 \\
Abr & $-0,67$ & 0,23 & 0,005 & $-0,49$ & 0,29 & 0,097 \\
May & $-0,70$ & 0,24 & 0,004 & $-0,29$ & 0,30 & 0,343 \\
Jun & $-0,58$ & 0,25 & 0,024 & $-0,34$ & 0,31 & 0,265 \\
Jul & $-0,77$ & 0,23 & 0,001 & $+0,14$ & 0,37 & 0,717 \\
Ago & $-0,65$ & 0,23 & 0,006 & $-0,35$ & 0,30 & 0,253 \\
Sep & $-0,60$ & 0,24 & 0,014 & $-0,25$ & 0,31 & 0,428 \\
Oct & $-0,54$ & 0,24 & 0,027 & $-0,41$ & 0,30 & 0,177 \\
Nov & $-0,65$ & 0,24 & 0,008 & $-0,50$ & 0,29 & 0,090 \\
Dic & $-0,79$ & 0,23 & 0,001 & $-0,38$ & 0,31 & 0,217 \\
\hline
\end{tabular}

1 Enero es el mes de referencia $(B=0)$, y por tanto su valor equivale al intercepto.

1 January is the reference month $(B=0)$ and therefore its value is the equivalent of the intercept

Tabla 2.- B-parámetros del efecto del mes en el número de capturas de piquituertos jóvenes capturados en dos localidades del Pirineo Navarro $(n=619$ días de muestreo).

Table 2.- $B$-parameter estimates of the effect of month on the number of captures of juvenile crossbills caught at two localities of the Pyrenean region of Navarre ( $n=619$ sampling days).

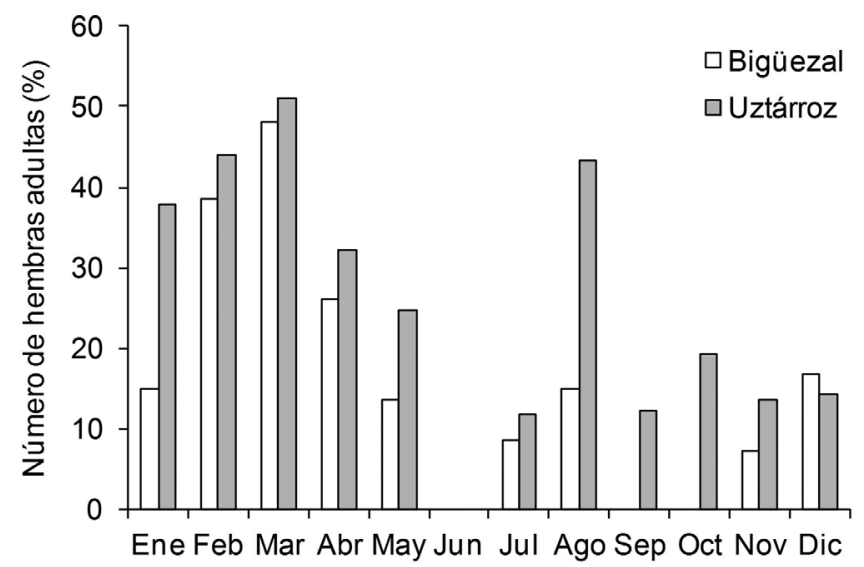

Fig.3.- Porcentaje de hembras adultas capturadas en reproducción activa (placas incubatrices código 2 y 3 , equivalentes al periodo de incubación).

Fig. 3.- Percentage of adult female birds caught as active breeders (brood patch score 2 and 3, equivalent to incubating period). 


\section{Discusión}

En el área de estudio la abundancia de piquituertos a lo largo del ciclo anual presenta un patrón fenológico particular. Concretamente, observamos un máximo de abundancia en el número de adultos, relativamente estable entre los meses de enero-abril, coincidiendo con el periodo de reproducción de la especie en la región (véase Fig. 3). En base a este patrón de abundancia, el promedio de capturas durante el periodo enero-abril podría ser un índice útil a la hora de determinar la abundancia de adultos durante el periodo de cría. Este índice, en todo caso, se aplicaría a la fracción de aves nidificantes durante el periodo de cría principal y no a los adultos que puedan reproducirse en verano (Alonso \& Arizaga, 2011). Esto se debe a que los piquituertos se reproducen en el momento de apertura de las piñas que, en el caso del pino silvestre, tiene lugar en invierno. La reproducción en verano (agosto-septiembre) afecta a un porcentaje bajo de la población y es irregular; posiblemente implica ejemplares locales y está vinculada a años de alta producción de semillas, si bien es un fenómeno poco estudiado (Alonso \& Arizaga, 2011).

Los patrones de abundancia fueron similares en las dos localidades estudiadas. En paralelo, el patrón fenológico de hembras en estado reproductor activo también fue similar entre las dos zonas de muestreo, con tan sólo ciertas variaciones locales. Esto significa que los resultados de nuestro estudio se podrían asumir a escala regional.

Tras el periodo de nidificación, la abundancia de los piquituertos desciende (en el caso de Uztárroz abruptamente), para permanecer baja hasta el final del año. Este descenso podría reflejar o bien un abandono de la zona (Alonso et al., 2016) o un cambio en el comportamiento de los piquituertos (Cramp \& Perrins, 1994). Las aves fueron capturadas en puntos que son utilizados para la ingesta de mineral (sales). Aunque desconocemos hasta qué punto las sales son un recurso difícil de encontrar en la zona, sí observamos que la necesidad de estas sales durante el periodo de cría atraería aves de ambos sexos a los dos puntos de muestreo. Al alimentarse casi exclusivamente de semillas de pino, los piquituertos requieren un gran aporte de agua y minerales a lo largo de todo el año (Cramp \& Perrins, 1994). La demanda de estos últimos aumenta considerablemente durante el periodo reproductor, ya que también son utilizados para la producción de huevos (Cramp \& Perrins, 1994). Por otro lado, la muda en la población estudiada tiene lugar en verano (Alonso \& Arizaga, 2011). La movilidad de las aves durante este periodo suele reducirse (Jenni \& Winkler, 1994), lo cual podría reflejarse en un descenso en el número capturas. En definitiva, aunque el método de captura pueda suponer un sesgo en el muestreo, el patrón estacional de abundancia es consistente durante el periodo de estudio.

En cuanto al patrón de abundancia de aves juveniles cabe destacar la existencia de un repunte de la abundancia en Bigüézal hacia el mes de julio, hecho que no observamos en Uztárroz. Esta falta de homogeneidad en el patrón de abundancia de jóvenes, podría apoyar la idea de la existencia de ciertas particularidades a nivel local. Situado más al sur, a una altitud más baja que Uztárroz y en la ruta que comunica los Pirineos con otras áreas de pinar situadas en el Sistema Ibérico, Bigüézal podría considerarse como una zona de trán- 
sito, propicia, tal vez, para la sedimentación de aves en dispersión. Así, un ejemplar con plumaje totalmente juvenil anillado en julio de 2002 en Bigüézal se recapturó en septiembre de 2006 en Falces (al sur de Navarra), situado a 60km.

En conclusión, la homogeneidad en el patrón de abundancia de aves adultas entre ambos puntos de muestreo sugiere que los resultados hallados a nivel local se podrían extrapolar a toda la región y, en consecuencia, utilizarse como referencia en estaciones de anillamiento orientadas a trabajar con la especie de estudio.

\section{Agradecimientos}

Este proyecto ha sido financiado por D. Alonso. El Gobierno de Navarra autorizó el anillamiento de aves. E. del Val proporcionó valiosos comentarios que contribuyeron a mejorar una primera versión del trabajo.

\section{Bibliografía}

- Alonso, D., Arizaga, J. 2011. Seasonal patterns of breeding, moulting, and body mass variation in Pyrenean Common Crossbills Loxia curvirostra curvirostra. Ring. Migr. 26: 64-70.

- Alonso, D., Arizaga, J., Meier, C. M., Liechti, F. 2016. Light-level geolocators confirm resident status of a Southern European Common Crossbill population. J. Ornithol. 1-7.

- Arizaga, J., Alonso, D., Edelaar, P. 2015. Stable isotopes in a southern European crossbill population indicate restricted movement between regions with different pine species. J. Zool. 295: 49-55.

- Arizaga, J., Alonso, D., Hobson, K. 2014. Disentangling the origin of crossbills using morphology and isotopic $(\delta 2 \mathrm{H})$ characters. Are southern European crossbills restricted to population-specific key resources? J. Ornithol. 155: 1027-1035.

- Arizaga, J., Crespo, A., Iraeta, A. 2013. Noticias EMAN, 1. Sociedad de Ciencias Aranzadi. Donostia.

- Bates, D., Maechler, M., Bolker, B., Walker, S. 2014. "Ime4: Linear mixed-effects models using Eigen and S4. R package version 1.1-7. Available from http://CRAN.R-project.org/ package $=$ Ime 4 .

- Borrás, A. Senar, J.C. 2003. Piquituerto común, Loxia curvirostra. En: Atlas de las aves reproductoras de España. Martí, R., Del Moral, J.C. (Ed.): 588-589. DGCN-SEO/BirdLife. Madrid.

- Cramp, S., Perrins, C.M. 1994. Handbook of the Birds of Europe, the Middle East and North Africa. Vol. 8. Oxford University Press. Oxford.

- Jenni, L., Winkler, R. 1994. Moult and ageing of European passerines. Academic Press. London. 
- Loidi, J., Báscones, J.C. 2006. Memoria del mapa de vegetación de Navarra. Gobierno de Navarra. Pamplona.

- Newton, I. 1972. Finches. Collins. London.

- Nur, N., Geupel, G.R., Ballard, G. 2000. The use of constant-effort mist-netting to monitor demographic processes in passerine birds: annual variation in survival, productivity and floaters. 3rd Partners in Flight workshop. Ogden. UT.

- Peach, W.J., Baillie, S.R., Balmer, D.E. 1998. Long-term changes in the abundance of passerines in Britain and Ireland as measured by constant effort mist-netting. Bird Study 45: 257275.

- Peach, W.J., Buckland, S. T., Baillie, S. R. 1996. The use of constant effort mist-netting to measure between-year changes in the abundance and productivity of common passerines. Bird Study 43: 142-156.

- Purroy, F.J. 1975. Evolución anual de la avifauna en un bosque mixto de frondosas y coníferas en Navarra. Ardeola 21: 669-697.

- R Core Team (2014). "R: A language and environment for statistical computing." Available from http://www.R-project.org.

- Ralph, C. J., Dunn, E. H. 2004. Monitoring bird populations using mist nets.

- Sánchez, A. 1991. Estructura y estacionalidad de las comunidades de aves de la Sierra de Gredos. Ardeola 38: 207-231.

- Senar, J.C., Borras, A., Cabrera, T., Cabrera, J. 1993. Testing for the relationship between coniferous crop stability and Common Crossbill residence. J. Field Ornithol. 64: 464-469.

- Svensson, L. 1996. Guía para la identificación de los paseriformes europeos. Sociedad Española de Ornitología. Madrid. 